\title{
GLOBAL REGULARITY OF THE TWO-DIMENSIONAL BOUSSINESQ EQUATIONS WITHOUT DIFFUSIVITY IN BOUNDED DOMAINS
}

\author{
DAOGUO ZHOU
}

\begin{abstract}
We address the well-posedness for the two-dimensional Boussinesq equations with zero diffusivity in bounded domains. We prove global in time regularity for rough initial data: the initial velocity has $\epsilon$ fractional derivatives in $L^{q}$ and the initial temperature is in $L^{q}$, for some $q>2$ and $\epsilon>0$ arbitrarily small.
\end{abstract}

\section{Introduction and Main Results}

In this paper, we study the initial-boundary value problem for the 2D Boussinesq equations with zero thermal diffusivity on an open bounded domain $\Omega \subset \mathbb{R}^{2}$ with smooth boundary $\partial \Omega$. The corresponding equations reads

$$
\left\{\begin{array}{l}
\partial_{t} u+u \cdot \nabla u-\nu \Delta u+\nabla p=\theta e_{2} \\
\partial_{t} \theta+u \cdot \nabla \theta=0 \\
\nabla \cdot u=0
\end{array}\right.
$$

where $u=\left(u_{1}, u_{2}\right)$ is the velocity vector field, $p$ is the pressure, $\theta$ is the temperature, $\nu>0$ is the constant viscosity, and $e_{2}=(0,1)$. This system is supplemented by the following initial and boundary conditions

$$
\left\{\begin{array}{l}
(u, \theta)(x, 0)=\left(u_{0}, \theta_{0}\right)(x), x \in \Omega, \\
\left.u(x, t)\right|_{\partial \Omega}=0 .
\end{array}\right.
$$

Here, we have imposed the mostly used no-slip conditions on the velocity, which assume that fluid particles are adherent to the boundary due to the positive viscosity.

The Boussinesq equations play an important role in modeling large scale atmospheric and oceanic flows [18, 22. In addition, the Boussinesq equations is closely related to Rayleigh-Benard convection [22]. From the mathematical view, the 2D Boussinesq equations serve as a simplified model of the 3D Euler and Navier-Stokes equations. In fact, we get the $2 \mathrm{D}$ Boussinesq equations when we analyze $3 \mathrm{D}$ axisymmetric swirling fluid in the Navier-Stokes framework. Better understanding of the 2D Boussinesq equations will undoubtedly shed light on the understanding of $3 \mathrm{D}$ flows $[19$.

Recently, the well-posedness of the 2D Boussinesq equations has attracted attention of many mathematicians, see [1]-44, 6]-11], [15, [17, [16, 20, 24, 25]. In particular, when $\Omega=\mathbb{R}^{2}$, the Cauchy problem of (1.1) has been well

2010 Mathematics Subject Classification. 35Q35, 76D05.

Key words and phrases. Global regularity, Boussinesq equations, Zero diffusivity, Bounded domain. 
studied. Hou and $\mathrm{Li}$ [9] and Chae [4] showed the global in time regularity for $\left(u_{0}, \theta_{0}\right) \in H^{3}\left(\mathbb{R}^{2}\right) \times H^{2}\left(\mathbb{R}^{2}\right)$. Kukavica, Wang, Ziane [13] obtained the global regularity for $\left(u_{0}, \theta_{0}\right) \in W^{1+s, q}\left(\mathbb{R}^{2}\right) \times W^{s, q}\left(\mathbb{R}^{2}\right)$ for $s \in(0,1), q \in[2, \infty)$ and $s q>2$. They also pointed out that the restriction $s q>2$ can be removed provided that the initial data have compact support or $\Omega=\mathbb{T}^{2}$. Abidi and Hmidi [1] proved the global existence for $\left(u_{0}, \theta_{0}\right) \in L^{2}\left(\mathbb{R}^{2}\right) \cap B_{\infty, 1}^{-1}\left(\mathbb{R}^{2}\right) \times L^{2}\left(\mathbb{R}^{2}\right)$. Danchin and Paicu [6] proved the uniqueness of weak solution for $\left(u_{0}, \theta_{0}\right) \in L^{2}\left(\mathbb{R}^{2}\right) \times L^{2}\left(\mathbb{R}^{2}\right)$.

In real world applications, fluids often move in bounded domains, where new phenomena such as the creation of vorticity on the boundary appears. In such case, the boundary effect requires a careful analysis. The initial-boundary value problem of (1.1)-(1.2) was first studied by Lai, Pan, and Zhao [15, who showed the global regularity for $\left(u_{0}, \theta_{0}\right) \in H^{3}(\Omega) \times H^{2}(\Omega)$. Later, Hu, Kukavica and Ziane [10] proved the global existence for initial data $\left(u_{0}, \theta_{0}\right) \in H^{2}(\Omega) \times H^{1}(\Omega)$. Recently, He [8] established the uniqueness of weak solution in the energy space $L^{2}(\Omega) \times L^{2}(\Omega)$.

In the current paper, we study further the initial-boundary value problem of system (1.1)-(1.2). We improve the previous results to the case of rough initial data $\left(u_{0}, \theta_{0}\right) \in D_{A_{q}}^{1-\frac{1}{p}, p}(\Omega) \times W^{s, q}(\Omega)$. Here, $D_{A_{q}}^{1-\frac{1}{p}, p}$ denotes some fractional domain of the Stokes operator whose elements have $2-\frac{2}{p}$ derivatives in $L^{q}$ (see Section 2 for the definition).

Before stating our main results, we define the function spaces in which existence is going to be shown.

Definition 1.1. For all $T>0, s \geq 0$ and $1<p, q<\infty$, we denote $M_{T}^{p, q, s}$ the set of triples $(u, p, \theta)$ such that

$$
\begin{gathered}
u \in C\left([0, T] ; D_{A_{q}}^{1-\frac{1}{p}, p}\right) \cap L^{p}\left(0, T ; W^{2, q} \cap W_{0}^{1, q}\right), \partial_{t} u \in L^{p}\left(0, T ; L^{q}\right), \\
p \in L^{p}\left(0, T ; W^{1, q}\right) \text { and } \int_{\Omega} p d x=0, \\
\theta \in C\left([0, T] ; W^{s, q}\right), \partial_{t} \theta \in L^{p}\left(0, T ; W^{-1, q}\right) .
\end{gathered}
$$

The corresponding norm is denoted by $\|\cdot\|_{M_{T}^{p, q, s}}$.

Our main results read as follows.

Theorem 1.2. Let $\Omega$ be a bounded domain in $\mathbb{R}^{2}$ with $C^{2+\epsilon}$ boundary. Let $p \in$ $(1, \infty), q \in(2, \infty)$ and $s \in[0,1]$. Let $u_{0} \in D_{A_{q}}^{1-\frac{1}{p}, p}$ and $\theta_{0} \in W^{s, q}$. Then system (1.1)-(1.2) has a unique global solution which belongs to $M_{T}^{p, q, s}$ for all $T>0$.

As a byproduct of the proof of Theorem 1.2, we get the global regularity for $\left(u_{0}, \theta_{0}\right) \in H^{1}(\Omega) \times H^{1}(\Omega)$.

Proposition 1.3. Let $\Omega$ be a bounded domain in $\mathbb{R}^{2}$ with $C^{2+\epsilon}$ boundary. Assume that $u_{0} \in H^{1}, \nabla \cdot u_{0}=0$ and $\theta_{0} \in H^{1}$. Then there exists a unique global solution to system (1.1)-(1.2) such that for all $T>0$

$$
\begin{gathered}
u \in L^{\infty}\left(0, T ; H^{1}\right) \cap L^{2}\left(0, T ; H^{2}\right), \nabla u \in L^{1}\left(0, T ; L^{\infty}\right), \\
\theta \in L^{\infty}\left(0, T ; H^{1}\right) .
\end{gathered}
$$

Remark 1.4. While preparing the manuscript, the author becomes to know that Proposition 1.3 was obtained very recently by Ju [12] independently. However, our method is completely different from that of Ju, which exploited Brezis-Gallouet type inequalities and spectral decomposition. 
Remark 1.5. The proof of Theorem 1.2 is based on the maximal regularity of the Stokes operator and some interpolation inequalities. Our method is elementary and can be carried over to the whole space case $\mathbb{R}^{2}$ without difficulty.

Remark 1.6. Since the "regularity index" $2-2 / p$ in Theorem 1.2 can be arbitrarily close to zero, our result improves the previous works of Lai et al. 15, Hu et al. [10] and Kukavica et al. [13] by requiring much less regularity for the initial data.

The rest of this paper is organized as follows. In Section 2, we recall the maximal regularity of Stokes equations as well as some elementary inequalities. In Section 3 , we present the detailed proofs of the main results.

\section{PRELIMINARIES}

\section{Notations:}

(1) Let $\Omega$ be a bounded domain in $\mathbb{R}^{2}$. For $1<q<\infty$, denote by $L_{\sigma}^{q}(\Omega)$ the completion in $L^{q}(\Omega)$ of the set of solenoidal vector-fields with coefficients in $C_{0}^{\infty}(\Omega)$. If $k$ is an integer, we denote by $W^{k, q}(\Omega)$ the set of $L^{q}(\Omega)$ functions whose derivatives up to order $k$ belong to $L^{q}(\Omega)$. For $s \in(0,1)$, the Sobolev space $W^{s, q}(\Omega)$ is defined as

$$
W^{s, q}(\Omega)=\left\{f \mid\|f\|_{W^{s, q}}=\left(\int_{\Omega} \int_{\Omega} \frac{|f(x)-f(y)|^{q}}{|x-y|^{s q+2}} d x d y\right)^{\frac{1}{q}}<\infty\right\} .
$$

(2) For $\alpha \in(0,2)$ and $1<p, q<\infty$, denote by $B_{q, p}^{\alpha}$ the Besov space which is defined as the real interpolation space between $L^{q}(\Omega)$ and $W^{m, q}(\Omega)(m>\alpha)$ :

$$
B_{q, r}^{\alpha}(\Omega)=\left(L^{q}(\Omega), W^{m, q}(\Omega)\right)_{\frac{\alpha}{m}, r}
$$

Denote by $\stackrel{\circ}{B}_{q, p}^{\alpha}$ the completion of $C_{0}^{\infty}(\Omega)$ in $B_{q, p}^{\alpha}$. See Adams and Fournier [3] for more about the Besov space.

(3) For $T>0$ and a function space $X$, denote by $L^{p}(0, T ; X)$ the set of Bochner measurable $X$-valued time dependent functions $f$ such that $t \rightarrow\|f\|_{X}$ belongs to $L^{p}(0, T)$.

First we give the definition of the fractional domains of the Stokes operator in $L^{q}$.

Definition 2.1. For $\alpha \in(0,1)$ and $s, q \in(1, \infty)$, we set

$$
\|u\|_{D_{A_{q}}^{\alpha, s}}=\|u\|_{L^{q}}+\left(\int_{0}^{\infty}\left\|t^{1-\alpha} A_{q} e^{-t A_{q}} u\right\|_{L^{q}}^{s} \frac{d t}{t}\right)^{\frac{1}{s}},
$$

where $A_{q}=-\mathbb{P} \Delta$ has domain $D\left(A_{q}\right)=W^{2, q}(\Omega) \cap W_{0}^{1, q}(\Omega) \cap L_{\sigma}^{q}(\Omega)$. Here, $\mathbb{P}$ denotes the Leray projector.

Roughly, the vector fields of $D_{A_{q}}^{\alpha, s}$ have $2 \alpha$ derivatives in $L^{q}$, are divergence-free, and vanish on $\partial \Omega$. In fact, we have the following imbedding (cf. Proposition 2.5 in Danchin [5]).

Lemma 2.2. $\stackrel{\circ}{B}_{q, s}^{2 \alpha} \cap L_{\sigma}^{q} \hookrightarrow D_{A_{q}}^{\alpha, s} \hookrightarrow B_{q, s}^{2 \alpha} \cap L_{\sigma}^{q}$, for $\alpha \in(0,1), 1<q, s<\infty$. Moreover, if $2 \alpha \leq \frac{1}{q}$, then the three spaces are the same (with equivalent norms).

We need the well-known Sobolev embedding, Ladyzhenskaya inequality and Gagliardo-Nirenberg inequality (see Adams and Fournier [3, Ladyzhenskaya 14] and Nirenberg [21]). 
Lemma 2.3. Let $\Omega \in \mathbb{R}^{2}$ be a bounded domain with $C^{2}$ boundary. Then the following embeddings and inequalities hold:

(1) $H^{1}(\Omega) \hookrightarrow L^{q}(\Omega)$, for all $q \in(1, \infty)$.

(2) $\|f\|_{L^{4}} \leq \sqrt{2}\|f\|_{L^{2}}^{\frac{1}{2}}\|\nabla f\|_{L^{2}}^{\frac{1}{2}}$, for $f \in H_{0}^{1}(\Omega)$.

(3) $\|\nabla u\|_{L^{\infty}} \leq C\left\|\nabla^{2} u\right\|_{L^{q}}^{\alpha}\|u\|_{L^{q}}^{1-\alpha}+C\|u\|_{L^{q}}$, for all $u \in W^{2, q}(\Omega)$, with $q \in$ $(2, \infty), \alpha=\frac{1}{2}+\frac{1}{q}$ and $C$ is a constant depending on $q, \Omega$.

We also need the following interpolation inequality (cf. Lemma 4.1 in Danchin [5]).

Lemma 2.4. Let $1<p, q<\infty$ satisfy $0<\frac{1}{2}-\frac{1}{q}<\frac{1}{p}$. The following inequality holds true:

$$
\|\nabla u\|_{L^{p}\left(0, T ; L^{\infty}\right)} \leq C T^{\frac{1}{2}-\frac{1}{q}}\|f\|_{L^{\infty}\left(0, T ; D_{A_{q}}^{\left.1-\frac{1}{p}\right)}\right.}^{1-\theta}\|f\|_{L^{p}\left(0, T ; W^{2, q}\right)}^{\theta},
$$

for $C=(p, q, \Omega)$ and $\frac{1-\theta}{p}=\frac{1}{2}-\frac{1}{q}$.

Now we recall the following standard result for linear transport equations.

Lemma 2.5. Let $\Omega$ be a Lipschitz domain of $\mathbb{R}^{2}$ and $u \in L^{1}\left(0, T ; W^{1, \infty}\right)$ such that $\operatorname{div} u=0$ and $u \cdot n=0$ on $\partial \Omega$. Let $a_{0} \in W^{s, q}$ with $q \in[1, \infty)$ and $s \in[0,1]$. Then the equation

$$
\left\{\begin{array}{l}
\partial_{t} a+u \cdot \nabla a=0, \\
\left.a(x, t)\right|_{t=0}=a_{0},
\end{array}\right.
$$

has a unique solution in $C\left([0, T] ; W^{s, q}\right)$. Moreover, the following estimate holds true for all $t \in[0, T]$

$$
\|a(t)\|_{W^{s, q}} \leq\left\|a_{0}\right\|_{W^{s, q}} e^{C \int_{0}^{t}\|\nabla v(\tau)\|_{L^{\infty} d \tau}},
$$

with $C=C(s, q)$. If in addition a belongs to $L^{p}$ for some $p \in[1, \infty]$ then for all $t \in[0, T]$

$$
\|a(t)\|_{L^{p}}=\left\|a_{0}\right\|_{L^{p}}
$$

The result for $s=0$ and $s=1$ is well-known (cf. Proposition 3.1 in Danchin [5])). The case $s \in(0,1)$ seems to be folklore, but I can not locate the proof. Here we provide a sketched proof.

Proof. We only establish a priori estimates and refer the reader to Desjardins [7] for the existence and uniqueness parts.

Denote by $\psi_{t}(x)$ the flow of $u$, which is defined by

$$
\partial_{t} \psi_{t}(x)=u\left(\psi_{t}(x), t\right),\left.\quad \psi_{t}(x)\right|_{t=0}=x \in \Omega,
$$

It follows that (2.1) has the formal solution

$$
a(x, t)=a_{0}\left(\psi_{t}^{-1}(x)\right) .
$$

From the assumptions $\operatorname{div} u=0$ and $u \in L^{1}\left(0, T ; W^{1, \infty}\right)$, we obtain

$$
\left|\operatorname{det} \psi_{t}(x)\right|=1 \text {, and }|x-y| \leq\left|\psi_{t}(x)-\psi_{t}(y)\right| e^{\int_{0}^{t}\|\nabla u(\tau)\|_{L} \infty d \tau} .
$$

See Chapter 4 in Majda 18 for more details. 
Using the definition of fractional Sobolev space and (2.2), we can compute $\|a(t)\|_{W^{s, q}}$ as follows:

$$
\begin{aligned}
\|a(t)\|_{W^{s, q}} & =\left(\int_{\Omega} \int_{\Omega} \frac{\left|a_{0}\left(\psi_{t}^{-1}(x)\right)-a_{0}\left(\psi_{t}^{-1}(y)\right)\right|^{q}}{|x-y|^{s q+2}} d x d y\right)^{\frac{1}{q}} \\
& =\left(\int_{\Omega} \int_{\Omega} \frac{\left|a_{0}(u)-a_{0}(v)\right|^{q}}{\left|\psi_{t}(u)-\psi_{t}(v)\right|^{s q+2}}\left|\operatorname{det} \nabla \psi_{t}(u) \| \operatorname{det} \psi_{t}(v)\right| d u d v\right)^{\frac{1}{q}} \\
& =\left(\int_{\Omega} \int_{\Omega} \frac{\left|a_{0}(u)-a_{0}(v)\right|^{q}}{|u-v|^{s q+2}}\left(\frac{|u-v|}{\left|\psi_{t}(u)-\psi_{t}(v)\right|}\right)^{s q+2} d u d v\right)^{\frac{1}{q}} \\
& \leq\left\|a_{0}\right\|_{W^{s, q}} e^{\left(s+\frac{2}{q}\right) \int_{0}^{t}\|\nabla u(\tau)\|_{L} \infty d \tau} .
\end{aligned}
$$

This completes the proof of Lemma 2.5

We conclude this section by recalling the maximal regularity of the Stokes equations (cf. Theorem 3.2 in Danchin [5] or Theorem 1.1 in Solonnikov [23]), which will be used in the proof of Theorem 1.2 .

Lemma 2.6. Let $\Omega$ be a bounded domain with a $C^{2+\epsilon}$ boundary in $\mathbb{R}^{2}$ and $1<$ $p, q<\infty$. Assume that $u_{0} \in D_{A_{q}}^{1-\frac{1}{p}, p}, f \in L^{p}\left(0, \infty ; L^{q}\right)$. Then the system

$$
\left\{\begin{array}{l}
\partial_{t} u-\nu \Delta u+\nabla p=f, \\
\nabla \cdot u=0, \\
\left.u(x, t)\right|_{\partial \Omega}=0,\left.u(x, t)\right|_{t=0}=u_{0},
\end{array}\right.
$$

has a unique solution $(u, p)$ satisfying the following inequality for all $T>0$ :

$$
\begin{aligned}
& \|u\|_{L^{\infty}\left(0, T ; D_{A_{q}}^{1-\frac{1}{p}, p}\right)}+\|u\|_{L^{p}\left(0, T ; W^{2, q}\right)}+\left\|\partial_{t} u\right\|_{L^{p}\left(0, T ; L^{q}\right)}+\|p\|_{L^{p}\left(0, T ; W^{1, q}\right)} \\
& \leq C\left(\left\|u_{0}\right\|_{D_{A_{q}}^{1-\frac{1}{p}, p}}+\|f\|_{L^{p}\left(0, T ; L^{q}\right)}\right)
\end{aligned}
$$

with $C=C(p, q, \nu, \Omega)$.

\section{PROOF OF MAIN RESULTS}

In this section, we prove Theorem 1.2 and Proposition 1.3 . To do so, we make two preparations. The first is a local existence result for system (1.1)-(1.2).

Lemma 3.1. Let the conditions in Theorem 1.2 hold. Then there exists a $T_{0}=$ $T_{0}\left(\left\|u_{0}\right\|_{D_{A_{q}}^{1-\frac{1}{p}, p}},\left\|\theta_{0}\right\|_{L^{q}}\right)>0$ such that system (1.1)-(1.2) has a unique solution in $M_{T_{0}}^{p, q, s}$.

Proof. The proof consists of several steps, including constructing the approximate solutions, obtaining the uniform local in time estimates, showing the convergence, and proving the uniqueness.

First step: Construction of approximate solutions. We initialize the construction of approximate solutions by smoothing out the initial data $\left(u_{0}, \theta_{0}\right)$ and get a sequence of smooth initial data $\left(u_{0}^{n}, \theta_{0}^{n}\right)_{n \in \mathbb{N}}$ which is bounded in $D_{A_{q}}^{1-\frac{1}{p}, p} \times L^{q}$. In addition, these smooth data belong to the Sobolev space $H^{3}$. Hence, applying the result of Lai, Pan, and Zhao [15] provides us a sequence of smooth global solutions 
$\left(u^{n}, p^{n}, \theta^{n}\right)_{n \in \mathbb{N}}$, which satisfy that $\left(u^{n}, \theta^{n}\right) \in C\left([0, \infty) ; H^{3}\right) \cap C^{1}\left([0, \infty) ; H^{2}\right)$ and $p^{n} \in C\left([0, \infty) ; H^{3}\right)$.

Second step: Uniform estimate for some small fixed time $T_{0}$. We aim at finding a positive time $T_{0}$ independent of $n$ for which $\left(u^{n}, p^{n}, \theta^{n}\right)_{n \in \mathbb{N}}$ is uniformly bounded in the space $M_{T_{0}}^{p, q, s}$.

Applying Lemma 2.5 to the temperature equation, we find that for all $t \geq 0$ and $s \in[0,1]$

$$
\left\|\theta^{n}\right\|_{L^{\infty}\left(0, t ; L^{q}\right)} \leq\left\|\theta_{0}\right\|_{L^{q}}
$$

and

$$
\left\|\theta^{n}\right\|_{L^{\infty}\left(0, t ; W^{s, q}\right)} \leq\left\|\theta_{0}\right\|_{W^{s, q}} e^{C \int_{0}^{t}\left\|\nabla u^{n}(\tau)\right\|_{L^{\infty} d \tau}} .
$$

Considering the velocity equation, we obtain

$$
\begin{aligned}
& \left\|u^{n}\right\|_{L^{\infty}\left(0, t ; D_{A_{q}}^{1-\frac{1}{p}, p}\right)}+\left\|\partial_{t} u^{n}\right\|_{L^{p}\left(0, t ; L^{q}\right)}+\left\|u^{n}\right\|_{L^{p}\left(0, t ; W^{2, q}\right)}+\left\|p^{n}\right\|_{L^{p}\left(0, t ; W^{1, q}\right)} \\
& \leq C\left(\left\|u_{0}\right\|_{D_{A_{q}}^{1-\frac{1}{p}, p}}+\left\|u^{n} \cdot \nabla u^{n}\right\|_{L^{p}\left(0, t ; L^{q}\right)}+\left\|\theta^{n}\right\|_{L^{p}\left(0, t ; L^{q}\right)}\right) \\
& \leq C\left(\left\|u_{0}\right\|_{D_{A_{q}}^{1-\frac{1}{p}, p}}+\left\|u^{n}\right\|_{L^{\infty}\left(0, t ; L^{q}\right)}\left\|\nabla u^{n}\right\|_{L^{p}\left(0, t ; L^{\infty}\right)}+\left\|\theta^{n}\right\|_{L^{p}\left(0, t ; L^{q}\right)}\right) .
\end{aligned}
$$

If $\frac{2}{p}+\frac{2}{q}>1$, Lemma 2.4 yields for $\theta=1-\frac{p}{2}\left(1-\frac{2}{q}\right)$

$$
\left\|\nabla u^{n}\right\|_{L^{p}\left(0, t ; L^{\infty}\right)} \leq C t^{\frac{1}{2}-\frac{1}{q}}\left\|u^{n}\right\|_{L^{p}\left(0, t ; W^{2, q}\right)}^{\theta}\left\|u^{n}\right\|_{L^{\infty}\left(0, t ; D_{A_{q}}^{1-\frac{1}{p}, p}\right)}^{1-\theta} .
$$

If $\frac{2}{p}+\frac{2}{q}<1$, we have $D_{A_{q}}^{1-\frac{1}{p}, p} \hookrightarrow W^{1, \infty}$ so that

$$
\left\|\nabla u^{n}\right\|_{L^{p}\left(0, t ; L^{\infty}\right)} \leq C t^{\frac{1}{p}}\left\|u^{n}\right\|_{L^{\infty}\left(0, t ; D_{A_{q}}^{1-\frac{1}{p}, p}\right)} .
$$

If $\frac{2}{p}+\frac{2}{q}=1$, applying Hölder's inequality, we arrive at the following inequality,

$$
\left\|u^{n} \cdot \nabla u^{n}\right\|_{L^{p}\left(0, t ; L^{q}\right)} \leq\left\|u^{n}\right\|_{L^{\infty}\left(0, t ; L^{q+}\right)}\left\|\nabla u^{n}\right\|_{L^{p}\left(0, t ; L^{\frac{q q^{+}}{q^{+}-q}}\right)},
$$

where $q^{+}$is slightly bigger than $q$. Noticing that $D_{A_{q}}^{1-\frac{1}{p}, p} \hookrightarrow W^{1, \frac{q q^{+}}{q^{+}-q}}$ and $D_{A_{q}}^{1-\frac{1}{p}, p} \hookrightarrow$ $L^{q^{+}}$, we eventually get

$$
\left\|u^{n} \cdot \nabla u^{n}\right\|_{L^{p}\left(0, t ; L^{q}\right)} \leq C t^{\frac{1}{p}}\left\|u^{n}\right\|_{L^{\infty}\left(0, t ; D_{A_{q}}^{1-\frac{1}{p}, p}\right)}^{2} .
$$

On the other hand, combining Hölder's inequality and (3.1), we have

$$
\left\|\theta^{n}\right\|_{L^{p}\left(0, t ; L^{q}\right)} \leq t^{\frac{1}{p}}\left\|\theta^{n}\right\|_{L^{\infty}\left(0, t ; L^{q}\right)} \leq t^{\frac{1}{p}}\left\|\theta_{0}\right\|_{L^{q}} .
$$

Define

$$
U^{n}(t)=\left\|u^{n}\right\|_{L^{\infty}\left(0, t ; D_{A_{q}}^{1-\frac{1}{p}, p}\right)}+\left\|\partial_{t} u^{n}\right\|_{L^{p}\left(0, t ; L^{q}\right)}+\left\|u^{n}\right\|_{L^{p}\left(0, t ; W^{2, q}\right)},
$$

and

$$
\left\|U^{0}\right\|=\left\|u_{0}\right\|_{D_{A_{q}}^{1-\frac{1}{p}, p}}+\left\|\theta_{0}\right\|_{L^{q}} .
$$


Inserting (3.4)-(3.8) into (3.3), we deduce that

$$
U^{n}(t) \leq C\left(\left\|u_{0}\right\|_{D_{A_{q}}^{1-\frac{1}{p}, p}}+\max \left(t^{\frac{1}{2}-\frac{1}{q}}, t^{\frac{1}{p}}\right)\left(U^{n}(t)\right)^{2}+t^{\frac{1}{p}}\left\|\theta_{0}\right\|_{L^{q}}\right) .
$$

Set

$$
T_{0}=\min \left\{1,\left(\frac{1}{4 C U_{0}}\right)^{p},\left(\frac{1}{4 C U_{0}}\right)^{\frac{2 q}{q-2}}\right\} .
$$

Direct computations show that for $t \in\left[0, T_{0}\right]$

$$
U^{n}(t) \leq I_{1}(t)=\frac{1-\sqrt{1-4 C \max \left(t^{\frac{1}{2}-\frac{1}{q}}, t^{\frac{1}{p}}\right) U_{0}}}{2 \max \left(t^{\frac{1}{2}-\frac{1}{q}}, t^{\frac{1}{p}}\right)} \leq 2 C U_{0},
$$

or

$$
U^{n}(t) \geq I_{2}(t)=\frac{1+\sqrt{1-4 C \max \left(t^{\frac{1}{2}-\frac{1}{q}}, t^{\frac{1}{p}}\right) U_{0}}}{2 \max \left(t^{\frac{1}{2}-\frac{1}{q}}, t^{\frac{1}{p}}\right)} \geq \frac{1}{2 \max \left(t^{\frac{1}{2}-\frac{1}{q}}, t^{\frac{1}{p}}\right)} .
$$

We show that (3.10) holds for $t \in\left[0, T_{0}\right]$. Since $u^{n} \in C\left([0, \infty) ; H^{3}\right)$ and $\lim _{t \rightarrow 0} I_{2}(t)=$ $\infty$, there exists some time $T_{1}>0$ such that (3.10) holds for $t \in\left[0, T_{1}\right]$. By contradiction, suppose that (3.10) does not hold for all $t \in\left[0, T_{0}\right]$, then there exists a first time $T_{2}>0$ such that (3.11) holds. It follows that $\lim _{t \rightarrow T_{2}-} U^{n}(t) \leq I_{1}\left(T_{2}\right)$ and $U^{n}\left(T_{2}\right) \geq I_{2}\left(T_{2}\right)$, which contradicts the fact $u^{n} \in C\left([0, \infty) ; H^{3}\right)$. Hence we have for all $t \in\left[0, T_{0}\right]$

$$
U^{n}(t) \leq 2 C U_{0} .
$$

Coming back to (3.2), noting that

$$
\int_{0}^{t}\left\|\nabla u^{n}(\tau)\right\|_{L^{\infty}} d \tau \leq \int_{0}^{t}\left\|u^{n}\right\|_{W^{2, q}} d \tau \leq t^{1-\frac{1}{p}}\left\|u^{n}\right\|_{L^{p}\left(0, T ; W^{2, q}\right)},
$$

we derive that

$$
\theta \in C\left([0, T] ; W^{s, q}\right), \quad \partial_{t} \theta^{n} \in L^{p}\left(0, T ; W^{-1, q}\right) .
$$

Third step: Passing to the limit. Since $\left(u^{n}, p^{n}, \theta^{n}\right)_{n \in \mathbb{N}}$ is uniformly bounded in the space $M_{T_{0}}^{p, q, s}$, applying Aubin-Lions lemma yields the solution to system (1.1)-(1.2) which belongs to $M_{T_{0}}^{p, q, s}$.

Fifth step: Uniqueness. The uniqueness is implied by the result in $\mathrm{He}$ 8, which says that the energy weak solution to system (1.1)-(1.2) is unique. This completes the proof of Lemma 3.2

The following lemma is the main ingredient of the proof of Theorem 1.2

Lemma 3.2. Let $\Omega$ be a bounded domain in $\mathbb{R}^{2}$ with $C^{2+\epsilon}$ boundary for some $\epsilon>0$. Let $p \in(1, \infty), q \in(2, \infty)$ and $s \in[0,1]$. Suppose that $u_{0} \in D_{A_{q}}^{1-\frac{1}{p}, p} \cap H^{1}$ and $\theta_{0} \in W^{s, q}$. Then system (1.1)-(1.2) has a unique global solution $(u, p, \theta)$ which belongs to $M_{T}^{p, q, s}$ for all $T>0$. Furthermore, we have

$$
u \in L^{\infty}\left(0, T ; H^{1}\right) \cap L^{2}\left(0, T ; H^{2}\right) .
$$

We divide the proof of Lemma 3.2 into three steps. First, we recall some elementary energy estimates. Next, we derive global $H^{1}$ estimate for the velocity. Finally, we use the maximal regularity of the Stokes operator to improve the regularity for both the velocity and the temperature. 


\section{Proof. Step 1 Energy Estimates.}

Let $T>0$ be any fixed given time. Reasoning as in Lemma 3.1, we get from the temperature equation for all $r \in[1, \infty)$

$$
\theta \in L^{\infty}\left(0, T ; L^{r}\right) .
$$

The basic energy estimate for the velocity equation yields that

$$
\frac{1}{2} \frac{d}{d t}\|u\|_{L^{2}}^{2}+\nu\|\nabla u\|_{L^{2}}^{2} \leq\|u\|_{L^{2}}\|\theta\|_{L^{2}} .
$$

Applying Gronwall's inequality, we have

$$
u \in L^{\infty}\left(0, T ; L^{2}\right) \cap L^{2}\left(0, T ; H^{1}\right) .
$$

Step $2 H^{1}$ Estimate for the Velocity.

Taking $L^{2}$-inner product of the velocity equation with $-\mathbb{P} \Delta u$, where $\mathbb{P}$ is the Leray projector. we deduce that

$$
\frac{1}{2} \frac{d}{d t}\|\nabla u\|_{L^{2}}^{2}+\nu\|\mathbb{P} \Delta u\|_{L^{2}}^{2}=\int_{\Omega} u \cdot \nabla u \mathbb{P} \Delta u d x-\int_{\Omega} \theta e_{2} \mathbb{P} \Delta u d x .
$$

We now estimate the right hand side of (3.16). For the first term, using Hölder's inequality, Gagliardo-Nirenberg's inequality and Young's inequality, we get

$$
\begin{aligned}
\left|\int_{\Omega} u \cdot \nabla u \mathbb{P} \Delta u d x\right| & \leq C\|u\|_{L^{4}}\|\nabla u\|_{L^{4}}\|\mathbb{P} \Delta u\|_{L^{2}} \\
& \leq C\|u\|_{L^{2}}^{\frac{1}{2}}\|\nabla u\|_{L^{2}}\|\mathbb{P} \Delta u\|_{L^{2}}^{\frac{3}{2}} \\
& \leq C\|u\|_{L^{2}}^{2}\|\nabla u\|_{L^{2}}^{4}+\frac{1}{4} \nu\|\mathbb{P} \Delta u\|_{L^{2}}^{2} .
\end{aligned}
$$

For the second term, it follows from the Cauchy-Schwarz inequality that

$$
\left|\int_{\Omega} \theta e_{2} \mathbb{P} \Delta u d x\right| \leq C\|\theta\|_{2}^{2}+\frac{1}{4} \nu\|\mathbb{P} \Delta u\|_{2}^{2}
$$

Substituting (3.17) and (3.18) into (3.16), we find that

$$
\frac{d}{d t}\|\nabla u\|_{L^{2}}^{2}+\nu\|\mathbb{P} \Delta u\|_{L^{2}}^{2} \leq C\left(\|u\|_{L^{2}}^{2}\|\nabla u\|_{L^{2}}^{2}\right)\|\nabla u\|_{L^{2}}^{2}+\|\theta\|_{L^{2}}^{2} .
$$

Then, from (3.14), (3.15) and Gronwall's inequality, we obtain

$$
u \in L^{\infty}\left(0, T ; H^{1}\right) \cap L^{2}\left(0, T ; H^{2}\right),
$$

which, by the Sobolev embedding (Lemma 2.3), implies that for all $q \in(2, \infty)$

$$
u \in L^{\infty}\left(0, T ; L^{q}\right) .
$$

\section{Step 3 Bootstrap Argument.}

We derive $W^{2, p}$ estimate for the velocity by the maximal regularity of the Stokes operator. To this end, we rewrite the velocity equation as follows,

$$
\begin{aligned}
\partial_{t} u-\nu \Delta u+\nabla p & =-u \cdot \nabla u+\theta e_{2}, \\
\nabla \cdot u & =0 .
\end{aligned}
$$

Using Lemma 2.6, we see that for $p \in(1, \infty), q \in(2, \infty)$,

$$
\begin{aligned}
& \|u\|_{L^{\infty}\left(0, T ; D_{A_{q}}^{1-\frac{1}{p}, p}\right)}+\left\|\partial_{t} u\right\|_{L^{p}\left(0, T ; L^{q}\right)}+\|u\|_{L^{p}\left(0, T ; W^{2, q}\right)}+\|p\|_{L^{p}\left(0, T ; W^{1, q}\right)} \\
& \leq C\left(\left\|u_{0}\right\|_{D_{A_{q}}^{1-\frac{1}{p}, p}}+\|u \cdot \nabla u\|_{L^{p}\left(0, T ; L^{q}\right)}+\|\theta\|_{L^{p}\left(0, T ; L^{q}\right)}\right) .
\end{aligned}
$$


We now estimate the term $\|u \cdot \nabla u\|_{L^{p}\left(0, T ; L^{q}\right)}$. Applying the interpolation inequality in Lemma 2.2, Hölder's inequality and Young's inequality, we find for any $\epsilon>0$

$$
\begin{aligned}
\|u \cdot \nabla u\|_{L^{p}\left(0, T ; L^{q}\right)} & \leq\|\nabla u\|_{L^{p}\left(0, T ; L^{\infty}\right)}\|u\|_{L^{\infty}\left(0, T ; L^{q}\right)} \\
& \leq C\left(\left\|\nabla^{2} u\right\|_{L^{p}\left(0, T ; L^{q}\right)}^{\alpha}\|u\|_{L^{p}\left(0, T ; L^{q}\right)}^{1-\alpha}+\|u\|_{L^{p}\left(0, T ; L^{q}\right)}\right)\|u\|_{L^{\infty}\left(0, T ; L^{q}\right)} \\
& \leq C\left(\epsilon\left\|\nabla^{2} u\right\|_{L^{p}\left(0, T ; L^{q}\right)}+C(\epsilon)\|u\|_{L^{p}\left(0, T ; L^{q}\right)}\right)\|u\|_{L^{\infty}\left(0, T ; L^{q}\right)} \\
& \leq C \epsilon\|u\|_{L^{\infty}\left(0, T ; L^{q}\right)}\left\|\nabla^{2} u\right\|_{L^{p}\left(0, T ; L^{q}\right)}+C(\epsilon) T^{\frac{1}{p}}\|u\|_{L^{\infty}\left(0, T ; L^{q}\right)}^{2} .
\end{aligned}
$$

Choosing $\epsilon$ small such that $C \epsilon\|u\|_{L^{\infty}\left(0, T ; L^{q}\right)} \leq \frac{1}{2 C}$, we get

$$
\|u \cdot \nabla u\|_{L^{p}\left(0, T ; L^{q}\right)} \leq \frac{1}{2 C}\left\|\nabla^{2} u\right\|_{L^{p}\left(0, T ; L^{q}\right)}+C T^{\frac{1}{p}}\|u\|_{L^{\infty}\left(0, T ; L^{q}\right)}^{2} .
$$

Substituting (3.22) into (3.21), together with (3.14) and (3.20), we deduce that

$$
\begin{aligned}
& \|u\|_{L^{\infty}\left(0, T ; D_{A_{q}}^{1-\frac{1}{p}, p}\right)}+\left\|\partial_{t} u\right\|_{L^{p}\left(0, T ; L^{q}\right)}+\|u\|_{L^{p}\left(0, T ; W^{2, q}\right)}+\|p\|_{L^{p}\left(0, T ; W^{1, q}\right)} \\
& \leq C\left(\left\|u_{0}\right\|_{D_{A_{q}}^{1-\frac{1}{p}, p}},\left\|u_{0}\right\|_{H^{1}},\left\|\theta_{0}\right\|_{L^{q}}, T\right) .
\end{aligned}
$$

Finally, reasoning similarly as in Lemma 3.1, we obtain estimate for the temperature in $L^{\infty}\left(0, T ; W^{s, q}\right)$. This completes the proof of Lemma 3.2

With Lemma 3.1 and 3.2 at hand, we are at a position to prove Theorem 1.2 If the initial velocity is smooth such that $u_{0} \in H^{1}$, then the proof is easy due to the global bound $u \in L^{\infty}\left(0, T ; H^{1}\right) \cap L^{2}\left(0, T ; H^{2}\right)$ for all $T>0$ (see Lemma 3.2). However, if the initial velocity is rough such that $u_{0} \notin H^{1}$, then the global $H^{1}$ bound for the velocity is absent, which makes it difficult to improve the regularity for the velocity by bootstrap argument. To solve this issue, we shall exploit the continuation argument due to Danchin (cf. Section 7 in [5]).

Proof of Theorem 1.2. We treat two cases $p \geq 2$ and $1<p<2$ differently.

(1) The Case of Smooth Data $p \geq 2$. Combining the imbedding $D_{A_{q}}^{1-\frac{1}{p}, p} \hookrightarrow$ $H^{1}$ (see Lemma 2.2) and Lemma 3.2 yields the result.

(2) The Case of Rough Data $1<p<2$. First, Lemma 3.1 gives us a local smooth solution $(u, p, \theta)$ with the initial data $\left(u_{0}, \theta_{0}\right)$. Let $T^{*} \in(0, \infty)$ be the existence time such that $(u, p, \theta)$ belongs to $M_{T^{*}}^{p, q, s}$. Then we shall prove that $T^{*}$ can be arbitrarily large by adapting the method of Danchin [5].

Since $u \in L^{p}\left(0, T^{*} ; W^{2, q}\right)$ and $\theta \in L^{\infty}\left(0, T^{*} ; W^{s, q}\right)$, there exists some $t_{0} \in\left(0, T^{*}\right)$ such that $u\left(t_{0}\right) \in W^{2, q} \cap L_{\sigma}^{q}$ and $\theta\left(t_{0}\right) \in W^{s, q}$. Noticing that $W^{2, q} \cap L_{\sigma}^{q} \hookrightarrow D_{A_{q}}^{1-\frac{1}{p}, p}$ (see Lemma 2.2), we obtain $u\left(t_{0}\right) \in D_{A_{q}}^{1-\frac{1}{p}, p} \cap H^{1}$. Due to Lemma 3.2. we can find a unique global smooth solution $(\tilde{u}, \tilde{\theta}, \tilde{p})$ to system (1.1)-(1.2) with initial data $\left(u\left(t_{0}\right), \theta\left(t_{0}\right)\right)$.

On the other hand, because the smooth solution to system (1.1)-(1.2) is unique, we get that $(u, p, \theta) \equiv(\tilde{u}, \tilde{\theta}, \tilde{p})$ on $\left(t_{0}, T^{*}\right)$. Thus, $(\tilde{u}, \tilde{\theta}, \tilde{p})$ is a global smooth continuation of $(u, p, \theta)$. This completes the proof of Theorem 1.2

Remark 3.3. The above argument does not give any information about the possible growth of the solution with respect to time. But it does work.

The proof of Proposition 1.3 is a direct consequence of Lemma 3.2 
Proof of Proposition 1.3. Choosing $p, q$ such that $\frac{1}{p}+\frac{1}{q}>1$ in Lemma 2.2, we have $H^{1} \cap L_{\sigma}^{2} \hookrightarrow D_{A_{q}}^{1-\frac{1}{p}, p}$. Then, it follows from Lemma 3.2 that

$$
u \in L^{\infty}\left(0, T ; H^{1}\right) \cap L^{2}\left(0, T ; H^{2}\right), \nabla u \in L^{1}\left(0, T ; L^{\infty}\right) .
$$

Applying Lemma 2.5 yields that

$$
\theta \in L^{\infty}\left(0, T ; H^{1}\right) .
$$

This completes the proof of Proposition 1.3 .

\section{ACKNOWLEDGEMENTS}

The author is supported in part by the National Natural Science Foundation of China (No. 11401176) and Doctor Fund of Henan Polytechnic University (No. B2012-110).

\section{REFERENCES}

1. H. Abidi, T. Hmidi, On the global well-posedness for Boussinesq system, J. Differential Equations 233 (2007) 199-220.

2. D. Adhikari, C. Cao, J. Wu, Global regularity results for the $2 \mathrm{D}$ Boussinesq equations with vertical dissipation, J. Differential Equations 251 (2011) 1637-1655.

3. R. Adams, J. Fournier, Sobolev spaces. Academic press, 2003.

4. D. Chae, Global regularity for the $2 \mathrm{D}$ Boussinesq equations with partial viscosity terms, Adv. Math. 203 (2006) 497-513.

5. R. Danchin, Density-dependent incompressible fluids in bounded domains, J. Math. Fluid Mech. 8 (2006) 333-381.

6. R. Danchin, M. Paicu, The Leray and Fujita-Kato theorems for the Boussinesq system with partial viscosity, Bull. Soc. Math. France 136 (2008) 261-309.

7. B. Desjardins, Linear transport equations with initial values in Sobolev spaces and application to the Navier-Stokes equations, Diff. Integral Eq. 10 (1997) 577-586.

8. L. He, Smoothing estimates of $2 \mathrm{~d}$ incompressible Navier-Stokes equations in bounded domains with applications, J. Funct. Anal. 262 (2012) 3430-3464.

9. T. Hou, C. Li, Global well-posedness of the viscous Boussinesq equations, Discrete Contin. Dyn. Syst. 12 (2005) 1-12.

10. W. Hu, I. Kukavica, M. Ziane, On the regularity for the Boussinesq equations in a bounded domain, J. Math. Phys. 54 (2013) 081507.

11. W. Hu, I. Kukavica, M. Ziane, Persistence of regularity for the viscous Boussinesq equations with zero diffusivity, Asymptot. Anal. 91 (2015) 111-124.

12. N. Ju, Global regularity and long-time behavior of the solutions to the $2 \mathrm{D}$ Boussinesq equations without diffusivity in a bounded domain, arXiv: 1508.06176, 2015.

13. I. Kukavica, F. Wang, M. Ziane, Persistence of regularity for solutions of the Boussinesq equations in Sobolev spaces, Adv. Diff. Eq. 21 (2016) 85-108.

14. O.A. Ladyzhenskaya, Mathematical Problems of the Dynamics of Viscous Incompressible Fluids, 2nd edition, Nauka, Moscow, 1970.

15. M. Lai, R. Pan, K. Zhao, Initial boundary value problem for two-dimensional viscous Boussinesq equations, Arch. Ration. Mech. Anal. 199 (2011) 739-760.

16. A. Larios, E. Lunasin, E. Titi, Global well-posedness for the 2D Boussinesq system with anisotropic viscosity and without heat diffusion, J. Differential Equations 255 (2013) 26362654 .

17. D. Li, X. Xu, Global well-posedness of an inviscid 2D Boussinesq system with nonlinear thermal diffusivity, Dyn. Partial Differ. Equ. 10 (2013) 255-265.

18. A. Majda, Introduction to PDEs and Waves for the Atmosphere and Ocean, Courant Lect. Notes Math., vol.9, AMS/CIMS, 2003.

19. A. Majda, A. Bertozzi, Vorticity and Incompressible Flow, Cambridge University Press, 2001.

20. C. Miao, X. Zheng, On the global well-posedness for the Boussinesq system with horizontal dissipation, Comm.Math.Phys. 321 (2013) 33-67. 
21. L. Nirenberg, On elliptic partial differential equations, Ann. Scuola Norm. Sup. Pisa 13.2 (1959): 115-162.

22. J. Pedlosky, Geophysical Fluid Dynamics, Springer-Verlag, New York, 1987.

23. V. Solonnikov, Estimates of solutions of the Stokes equations in SL Sobolev spaces with a mixed norm, J. Math. Sci 123.6 (2004): 4637-4653.

24. Y. Sun, Z. Zhang, Global regularity for the initial-boundary value problem of the 2-D Boussinesq system with variable viscosity and thermal diffusivity, J. Differential Equations 255 (2013) 1069-1085.

25. C. Wang, Z. Zhang, Global well-posedness for the 2-D Boussinesq system with the temperature-dependent viscosity and thermal diffusivity, Adv. Math. 228 (2011) 43-62.

School of Mathematics and Information Science, Henan Polytechnic University, JiaOzuo, Henan 454000, China

E-mail address: daoguozhou@hpu.edu.cn 